\title{
Developments in cell biology for quantitative immunoelectron microscopy based on thin sections: a review
}

\author{
Terry M. Mayhew $\cdot$ John M. Lucocq
}

Accepted: 26 May 2008/Published online: 14 June 2008

(C) Springer-Verlag 2008

\begin{abstract}
Quantitative immunoelectron microscopy uses ultrathin sections and gold particle labelling to determine distributions of molecules across cell compartments. Here, we review a portfolio of new methods for comparing labelling distributions between different compartments in one study group (method 1 ) and between the same compartments in two or more groups (method 2). Specimen samples are selected unbiasedly and then observed and expected distributions of gold particles are estimated and compared by appropriate statistical procedures. The methods can be used to analyse gold label distributed between volume-occupying (organelle) and surface-occupying (membrane) compartments, but in method 1, membranes must be treated as organelles. With method 1 , gold counts are combined with stereological estimators of compartment size to determine labelling density (LD). For volume-occupiers, LD can be expressed simply as golds per test point and, for surfaceoccupiers, as golds per test line intersection. Expected distributions are generated by randomly assigning gold particles to compartments and expressing observed/expected counts as a relative labelling index (RLI). Preferentially-labelled compartments are identified from their RLI values and by Chi-squared analysis of observed and expected distributions. For method 2, the raw gold particle counts distributed between compartments are simply compared across groups
\end{abstract}

\section{T. M. Mayhew $(\bowtie)$}

Centre for Integrated Systems Biology and Medicine,

School of Biomedical Sciences, Queen's Medical Centre,

E Floor, University of Nottingham, Nottingham NG7 2UH, UK

e-mail: terry.mayhew@nottingham.ac.uk

\section{J. M. Lucocq}

Division of Cell Biology and Immunology,

Wellcome Trust Biocentre, College of Life Sciences,

University of Dundee, Dundee, UK by contingency table and Chi-squared analysis. This identifies the main compartments responsible for the differences between group distributions. Finally, we discuss labelling efficiency (the number of gold particles per target molecule) and describe how it can be estimated for volume- or surfaceoccupiers by combining stereological data with biochemical determinations.

Keywords Quantitative immunoelectron microscopy . Relative labelling index · Labelling density · Contingency table and Chi-squared analysis . Labelling efficiency

\section{Introduction}

Cell biology is concerned with characterising the structure and composition of cells from the molecular to the microscopical level so as to understand better their growth, differentiation and activity. Transmission electron microscopy (TEM) plays an important role in this field by providing ways of quantifying structures and assessing the spatial distributions of molecules within ultrathin sections. Recently, efficient stereological methods for estimating cell and organelle composition at the ultrastructural level have been reviewed (Nyengaard and Gundersen 2006; Ochs 2006). The present article concerns itself with an important tool of cell biologists, immunoelectron microscopy, by which interesting molecules (often, but not exclusively, protein antigens) can be mapped at high resolution by applying a set of affinity reagents to sections and locating them with suitable markers (often colloidal gold conjugates). Crucially, particulate gold markers provide a digital signal that can be quantified easily and accurately (Griffiths 1993; Skepper 2000; Bendayan 2001). Moreover, 
recent developments have provided more robust sampling, stereological estimation and statistical procedures, which allow investigators to assess both the distribution and concentration of digital signals over cellular compartments (Lucocq 1994; Griffiths et al. 2001; Mayhew et al. 2002, 2003, 2004; Lucocq 2003, 2006, 2008; Lucocq et al. 2004; Mayhew and Desoye 2004; Razga and Nyengaard 2006, 2007; Mayhew 2007a; Mayhew and Lucocq 2008).

A key question addressed by the new methods is how to identify compartments that show preferential labelling. This is important for identifying compartments with the highest concentrations of interesting molecules and may also be of value in preliminary studies (see below and Fig. 1). A useful approach is to estimate a relative labelling index (RLI). This relates the observed numbers of gold particles over a set of compartments (within a given cell or cell system) to the expected numbers obtained when the same particles are distributed across compartments solely on the basis of compartment sizes. In essence, RLI indicates the degree to which a compartment is preferentially labelled in comparison to the theoretical situation of random labelling. RLI may be estimated directly or via labelling density (LD) values (Griffiths 1993; Mayhew et al. 2002, 2003, 2004; Mayhew 2007a). On ultrathin TEM sections, the LD of a compartment can be expressed as the numbers of gold particles in a profile area (organelles) or along a trace length (membranes). More simply, LD can be estimated as the number of particles per stereological point count (organelles) or per intersection count (membranes). The RLI approach is most easily applied when labelled antigens are

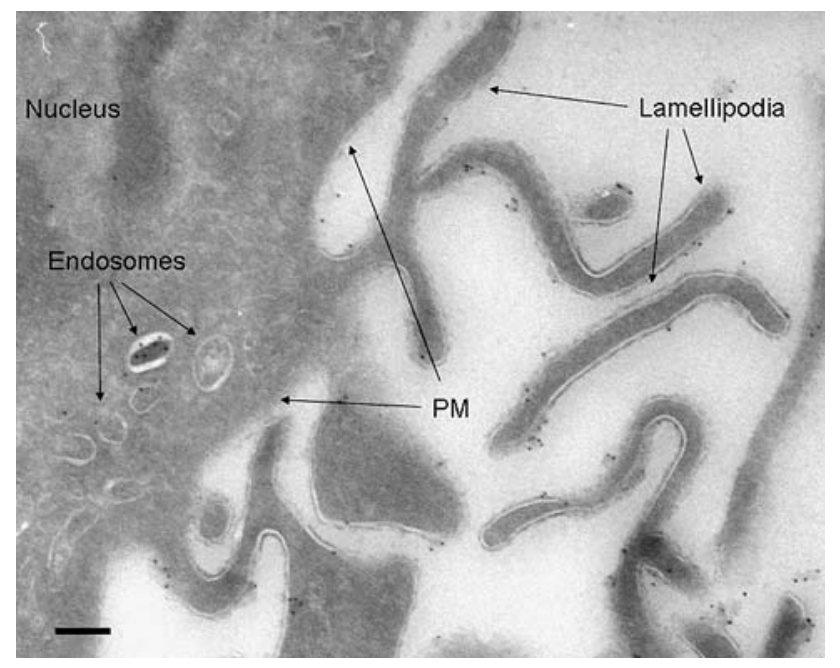

Fig. 1 Localisation of a viral receptor in a mouse dendritic cell. In this preliminary study, gold labelling appears concentrated in cell surface extensions called lamellipodia and peripheral profiles corresponding to tubular endosomes. Low amounts of label appear in the nucleus and at the plasma membrane (PM). Identification of compartments harbouring preferential labelling could be sought using method 1 and further dilutions of the antibody compared using method 2 (see text for details). Bar $100 \mathrm{~nm}$ localized in either organelle or membrane compartments rather than in a mixture of the two (Mayhew et al. 2002, 2003). However, it is possible to adapt the organelle-based model to embrace membrane compartments (Slot et al. 1991), thereby allowing analysis of molecules present in both, or translocating between, membranes and organelles. Methods for achieving this are currently under investigation (Mayhew 2007b; Mayhew and Lucocq 2008).

A second key question is how to compare shifts in labelling patterns between compartments in different groups of cells (e.g. control vs. experimental, or groups with different concentrations of antibody in preliminary labelling experiments). In such instances, it is not necessary to estimate compartment sizes or to calculate RLI. Instead, raw gold particle counts can be analysed and differences between distributions can be detected. Moreover, this approach can be used directly to study antigens localized in both organelles and membranes or those that translocate from one to the other.

This article focuses on these new methods and also reviews other recent techniques for quantifying gold labelling. Appropriate preliminary technical issues regarding the preparation and use of antibodies and gold probes, as well as the necessary tissue preparation and ultrathin sectioning procedures for TEM have been reviewed elsewhere (Griffiths 1993; Lucocq 1993; Maunsbach and Afzelius 1999; Skepper 2000; Bendayan 2001; Zuber et al 2005; Sosinsky et al. 2007).

\section{Overview of procedures}

Method 1: to compare the distributions of gold particles between different compartments within a cell or cell system

(1) Choose an appropriate set of compartments to suit the study aim (see "Additional guidance notes" below). For technical reasons, it is prudent to include those compartments which are known (or likely) to be labelled as well as others which are not. For statistical reasons, the number of compartments should be restricted so as to provide an acceptable balance between precision of localization (determined by the number of selected compartments) and precision of estimation (determined by the number of gold particles associated with each compartment or found in the cell as a whole).

(2) Design a multistage, preferably systematic uniform random (SUR), sampling scheme so as to ensure unbiased selection of section locations (and, if necessary, orientations) within the organ, tissue or cell preparation. This procedure will generate a set of microscopical fields suitable for analysis. 
(3) On the images of microscopical fields representing each group of cells, count gold particles associated with the selected compartments and construct an observed numerical frequency distribution of this labelling.

(4) If the compartments are volume occupiers (e.g. endosomes, mitochondria, nucleus, cytosol), generate an expected distribution by superimposing a lattice of test points on the microscopical images and count points associated with the profile areas of selected compartments. For each compartment, and for the whole cell, express LD as the number of gold particles per test point. In a similar fashion, if the compartments are all surface occupiers (membranes), generate an expected distribution by superimposing a lattice of test lines on the images and counting the intersections which these lines make with the membrane traces. LD for each compartment, and for the whole cell, is then calculated as the number of gold particles per test intersection. For each compartment, RLI $=\mathrm{LD}_{\text {comp }} /$ $\mathrm{LD}_{\text {cell. }}$. Mixtures of organelles and membranes can be accommodated by treating membranes (surface-occupying) as organelles (volume-occupying) and defining them with the aid of an acceptance zone (see below and Mayhew and Lucocq 2008).

(5) Compare observed numbers of gold particles on compartments with the predicted numbers of golds (derived from the observed frequencies of point or intersection counts). By means of a two-sample Chisquared $\left(\chi^{2}\right)$ analysis with two columns (observed and expected gold counts) and $c$ compartments (arranged in rows), compare the two distributions, calculate total and partial (compartmental) $\chi^{2}$ values, and determine whether to accept or reject the null hypothesis (of no difference between distributions) for $c-1$ degrees of freedom. For practical statistical reasons, compartments associated with 1-5 predicted gold particles should comprise no more than $20 \%$ of the identified compartments. Examination of the total $\chi^{2}$ value will indicate whether the gold labelling distributions are different. If the observed and expected distributions are different, examining the partial $\chi^{2}$ values will identify those compartments which are mainly responsible for that difference. A convenient arbitrary cut-off is a partial $\chi^{2}$ value accounting for $10 \%$ or more of total $\chi^{2}$.

Method 2: to compare the observed numbers of gold particles on compartments in different cell groups

(1)-(3). The steps here are as detailed above for method 1 . They include the following: (1) choosing a set of compartments appropriate to the study aim, (2) designing a multistage SUR sampling scheme to generate sectional images, and (3) using images to count gold particles associated with the selected compartments and constructing an observed numerical frequency distribution of the gold labelling.

(4) There is no need to take account of compartment size, and the observed gold counts can be analysed by means of a contingency table analysis with $g$ groups (arranged in columns) and $c$ compartments (arranged in rows). This analysis will generate predicted gold particles and, hence, partial $\chi^{2}$ values, for each group and each compartment. Again, there should be 1-5 predicted golds in each compartment. Examination of the total $\chi^{2}$ value, for $(g-1) \times(c-1)$ degrees of freedom, will indicate whether the gold labelling distributions are different. If they are different, examining the partial $\chi^{2}$ values (using the $10 \%$ cut-off) will identify the compartments which account for most of the between-group difference.

\section{Additional guidance notes}

\section{Defining cellular compartments}

To satisfy statistical requirements, it is wise to include in analyses not only the main compartments of interest (those thought to be labelled) but also other compartments not of individual interest (considered to be unlabelled or to show only background labelling). Decisions about the number of compartments to include will be influenced by several factors. For example, compartments not of primary interest may be brought together as a single artificial composite compartment (Mayhew et al. 2002). In addition, a compromise must be struck between the number of compartments selected (which determines the localization resolution) and the variability of gold particle counts within each compartment (which determines the precision of estimation). Generally speaking, the greater the number of compartments, the greater the resolution (or more precise the intracellular localization), but also the lower the estimation precision (which is related to $1 / \sqrt{ } n$ where $n$ is the number of gold particles within a given compartment), especially for infrequent, small or poorly-labelled compartments.

Comparing the distribution patterns of gold labelling in different groups of cells involves calculating expected gold particles in each compartment. For statistical testing to be accurate, it is preferable that no expected value should be less than 1 and no more than $20 \%$ of expected values should be less than 5. If these criteria are not met, it will be advisable to reduce the number of compartments (by omission or by combination) or to count more gold particles. 
An effective and unbiased way of choosing which compartments to include is to systematically sample 1-2 labelled grids, count 100-200 gold particles and identify the compartments with which they are associated (Lucocq et al. 2004). We also recommend defining at least 3 and no more than 12 compartments within a cell (Mayhew et al. 2002) and, when comparing different groups of cells, to count approximately equal total numbers of gold particles per cell group (Mayhew and Desoye 2004; Mayhew et al. 2004).

\section{Multistage random sampling}

Multistage random sampling of specimens (from selection of organs, tissues or cell cultures at the highest stage to selection of microscopical fields of view at the lowest) is critically important (Mayhew et al. 2002, 2003; Lucocq 2003, 2006, 2008; Lucocq et al. 2004; Mayhew 2008). Random sampling at each stage affords every part of the specimen the same chance of being selected and this is important regardless of the nature of the compartments (organelles, membranes, cytoskeletal elements) being investigated. It can also allow every orientation of the specimen the same chance of being selected (Baddeley et al. 1986; Mattfeldt et al. 1990; Nyengaard and Gundersen 1992). Indeed, the combination of random location with random orientation is necessary when dealing with membrane compartments or mixtures of organelles and membranes. SUR sampling tends to be more efficient than simple random sampling. With SUR sampling, the position and orientation of the first item can be selected at random and a pre-determined pattern (the sampling interval) dictates the positions and orientations of other items (Gundersen and Jensen 1987; Mayhew 1991, 2008; Gundersen et al. 1999).

Once cells have been sampled randomly, and decisions made about which compartments to include, image resolution on microscopical fields of view should be sufficient to allow identification of compartments and gold particles. It is then a relatively simple matter to count gold particles associated with each compartment. This can be achieved by recording fields digitally or photographically but a much more efficient method is to scan labelled sections at the TEM (Lucocq 2003, 2006, 2008; Lucocq et al. 2004). If this is undertaken as a pilot study in order to judge where the bulk of the labelled antigen resides, the results may be presented as a percentage frequency distribution. Recent examples of this sort of approach (Lucocq et al. 2004) have been published elsewhere (Coene et al. 2005; Young et al. 2005; Vasile et al. 2006; Hundorfean et al. 2007; Nithipongvanitch et al. 2007; Tomás et al. 2007). In contrast, for definitive studies using the present methods to compare particle distributions between compartments or to compare distribution patterns in different cell groups, it is important that raw counts are given as numerical frequency distributions and not converted into percentage frequency distributions. This constraint is necessary to meet the requirements for statistical comparisons.

\section{Practical details and examples}

Method 1: to compare the distributions of gold particles between different compartments within a cell or cell system

The objective is to test whether the observed distribution of gold particles between compartments within a cell is random. If not, then some compartments must be preferentially labelled. One way of testing for randomness in the distribution of gold particles is to construct the random (expected) distribution and then compare this to the observed distribution.

As an illustration, consider the localization of the proteolytic enzyme, m-calpain, in skeletal muscle. In a recent study of meat tenderization, Borjigin et al. (2006) examined the distribution of m-calpain in bovine serratus ventralis muscle immediately after thawing and after periods of post-mortem conditioning by storage at $2-4{ }^{\circ} \mathrm{C}$. Though the published data were confined to $\mathrm{LD}$ values (expressed as numbers of gold particles per $\mu \mathrm{m}^{2}$ ) in two different regions of sarcomeres (the I-band/Z-disk and Aband), we have modified their data to allow for the observation that the A-band region accounts for roughly $69 \%$ of the length of a sarcomere. Consequently, 69 out of every 100 test points randomly positioned within sarcomeres are expected to fall in this region. With these estimates, and the observed LD values (Borjigin et al. 2006), a dataset for control (thawed) muscle is summarised in Table 1.

The total points on the sarcomere (100) and the observed total number of gold particles (572) are used to calculate the expected gold particles for each region. For example, the expected number of gold particles for the I-band/Z-disk region is equal to $31 \times 572 / 100=177.3$. The benefit of this calculation is that we now have a direct measure of the degree to which this region of the sarcomere is labelled in comparison to random labelling. This relative labelling index $\left(\mathrm{RLI}_{\mathrm{IZ}}\right)$ is calculated by dividing observed gold particles by expected gold particles: $\mathrm{RLI}_{\mathrm{IZ}}=264 / 177.3=$ 1.49 approximately. Since $\mathrm{RLI}_{\mathrm{IZ}}$ is $>1$, it appears that this sarcomere region exhibits greater labelling than might be expected for a purely random deposition of gold particles. The corresponding partial $\chi^{2}$ for any compartment is calculated from observed $\left(N_{\text {obs }}\right)$ and expected $\left(N_{\text {exp }}\right)$ gold counts as 
Table 1 Empirical dataset illustrating method 1

\begin{tabular}{llllllr}
\hline Sarcomere regions & $\begin{array}{l}\text { Gold number, } \\
N_{\text {obs }}\end{array}$ & $\begin{array}{l}\text { Test point } \\
\text { number, } P\end{array}$ & $\begin{array}{l}\text { Expected } \\
\text { number, } N_{\text {exp }}\end{array}$ & $\begin{array}{l}\text { Labelling } \\
\text { density } \\
\text { LD }=N_{\text {obs }} / P\end{array}$ & $\begin{array}{l}\text { Relative labelling } \\
\text { index, } \\
\text { RLI }=\mathrm{LD}_{\text {comp }} / L_{\text {tot }}\end{array}$ \\
\hline I-band/Z-disk & 264 & 31 & 177.3 & 8.516 & 1.49 & $42.40(69.0 \%)$ \\
A-band & 308 & 69 & 394.7 & 4.464 & 0.78 & $19.04(31.0 \%)$ \\
Column totals & 572 & 100 & 572 & 5.720 & 1.00 & $61.44(100 \%)$ \\
\hline
\end{tabular}

Localization of m-calpain (marked by $10 \mathrm{~nm}$ gold particles) and random test points in two main sarcomere regions of normal bovine serratus ventralis muscle (see text and Borjigin et al. 2006). For total $\chi^{2}=61.44$ and 1 degree of freedom $(2-1$ columns $\times 2-1$ regions), $P<0.001$, so the distribution pattern of gold-labelled calpain is significantly different from random. The I-band/Z-disk region is preferentially labelled (RLI $=1.49$ and partial $\chi^{2}$ accounts for $69 \%$ of total $\chi^{2}$ )

$\chi^{2}=\left(N_{\mathrm{obs}}-N_{\mathrm{exp}}\right)^{2} / N_{\exp }$

which, for the I-band/Z-disk region, equates to (264 $177.3)^{2} / 177.3=42.40$ approximately. By the same argument, the partial $\chi^{2}$ for the A-band is 19.04 and total $\chi^{2}$ is 61.44 (Table 1). With 1 degree of freedom (given by $2-1$ columns $\times 2-1$ regions), the total $\chi^{2}$ gives a probability level of $P<0.001$ and so the null hypothesis of no difference from random labelling must be rejected.

Since the observed and expected distributions have been shown to be different, the criteria for deciding on preferential labelling of a compartment must be invoked. These are twofold: first, the value of RLI must be greater than 1 and, second, the partial $\chi^{2}$ value must account for a significant proportion (say $10 \%$ or more) of the total. On these grounds, the sarcomeres do display preferential labelling at the I-band/Z-disk region (Table 1).

Data generated for 14-day conditioned bovine muscle (Borjigin et al. 2006) reveal a similar non-random labelling pattern with the I-band/Z-disk region being preferentially labelled (Table 2). However, it is important to note that, although the analyses indicate that labelling distributions in both groups are non-random, this does not mean that the non-random pattern is the same in those groups. Indeed, analysis of the distributions between groups indicates that there is a shift in labelling between regions following conditioning for 14 days (see below).

For statistical testing by $\chi^{2}$ analysis, it is important to include a mix of labelled and unlabelled compartments, especially if the labelled compartments have similar RLI values (Mayhew et al. 2002). Chi-squared analysis also imposes minimal conditions regarding the numbers of expected gold particles on individual compartments. For example, it is recommended that no more than $20 \%$, and preferably none, of the compartments should have less than five expected gold particles (Mayhew and Desoye 2004; Mayhew et al. 2004) This may influence the choice of compartments or the numbers of observed gold particles to be counted. For instance, if it is desirable to identify separately a rare or poorly-labelled compartment, more effort will be required in counting gold particles associated with it. If the compartment is not of individual interest, it would be sensible simply to merge that compartment into some larger compartment such as "residuum" or "rest of cell".

An alternative way of testing for non-random labelling of compartments within a cell is to compare $L_{\text {comp }}$ for each compartment with $\mathrm{LD}_{\text {cell }}$ of the cell as a whole. By convention, LD is expressed as the number of gold particles per area of organelle profile or per length of membrane trace (Griffiths 1993; Mayhew et al. 2002). However, a more efficient approach is to express LD as the number of gold particles per test probe hit (i.e. per point for organelles or per test line intersection for membranes, Mayhew et al. 2003).

If all compartments within the cell were labelled randomly, we would expect them to display the same LD value. Therefore, $\mathrm{LD}_{\text {cell }}$ provides a very useful internal

Table 2 Empirical dataset illustrating method 1

\begin{tabular}{lcccccr}
\hline $\begin{array}{l}\text { Sarcomere } \\
\text { regions }\end{array}$ & $\begin{array}{l}\text { Gold number, } \\
N_{\text {obs }}\end{array}$ & $\begin{array}{l}\text { Test point } \\
\text { number, } P\end{array}$ & $\begin{array}{l}\text { Expected } \\
\text { number, } N_{\text {exp }}\end{array}$ & LD $=N_{\text {obs }} / P$ & RLI $=\mathrm{LD}_{\text {comp }} / \mathrm{LD}_{\text {tot }}$ & $\chi^{2}$ values $(\%)$ \\
\hline I-band/Z-disk & 616 & 31 & 479.9 & 19.871 & 1.28 & $38.60(69.0 \%)$ \\
A-band & 932 & 69 & $1,068.1$ & 13.507 & 0.87 & $17.34(31.0 \%)$ \\
Column totals & 1,548 & 100 & 1,548 & 15.480 & 1.00 & $55.94(100 \%)$ \\
\hline
\end{tabular}

Localization of m-calpain (marked by $10 \mathrm{~nm}$ gold particles) and random test points in two main sarcomere regions of bovine serratus ventralis muscle after post-mortem conditioning by storage at $2-4^{\circ} \mathrm{C}$ (see text and Borjigin et al. 2006). For total $\chi^{2}=55.94$ and 1 degree of freedom, $P<$ 0.001 . Again, the distribution of gold-labelled calpain is significantly different from random and the I-band/Z-disk region is preferentially labelled (RLI $=1.28$ and partial $\chi^{2}$ accounts for $69 \%$ of total $\chi^{2}$ ) 
reference and, in fact, the RLI of a given compartment can be calculated using the equation

$\mathrm{RLI}_{\text {comp }}=\mathrm{LD}_{\text {comp }} / \mathrm{LD}_{\text {cell }}$.

Tables 1 and 2 also provide LD values for each sarcomere region in bovine muscle to illustrate how RLI values for each region may be obtained by this indirect approach.

Since methods based on analysis of RLI or LD were first introduced (Mayhew et al. 2002, 2003, 2004; Mayhew and Desoye 2004), they have been applied to localize a variety of antigens in diverse cells and tissues (Ochs et al. 2002; Cernadas et al. 2003; Mironov et al. 2003; Bennett et al. 2004; Kweon et al. 2004; Mazzone et al. 2004; Wu et al. 2004; Fehrenbach et al. 2005; Potolicchio et al. 2005; Schmiedl et al. 2005; Signoret et al. 2005; Touret et al. 2005; Vancova et al. 2005; Young et al. 2005; Zhang et al. 2005; Li et al. 2006; Lopes et al. 2006; Vasile et al. 2006; Welsch et al. 2006; Davey et al. 2007; Driskell et al. 2007; Jacob et al. 2007; Tomás et al. 2007; Wilczynski et al. 2008). An unusual variant of the methods was applied by Fujii et al. (2003). These authors expressed labelling intensity in different groups of cells by multiplying RLI by LD for each compartment to obtain a so-called comparative labelling index. Because relative RLI and LD values show proportionality (Mayhew et al. 2002), this index is tantamount to exaggerating RLI differences by squaring them. The variant is essentially redundant.

Integrating data from membranes and organelles

As originally presented, the methods based on RLI and LD estimates deal effectively with between-compartment labelling differences when all compartments belong to the same category, e.g. they are all organelles or all membranes. However, some molecules translocate from membrane to vesicular or nuclear compartments (or vice versa) and so more recent developments have tried to provide LD estimators which treat all compartments in the same manner (Mayhew 2007b; Mayhew and Lucocq 2008). The steps in this process are as follows:

\section{Step 1: identify an acceptance zone}

The central problem in comparing gold-labelling distributions for surface- and volume-occupying compartments is calculating the expected distribution of gold particles for both types of structure. In theory, the expected distribution is generated by randomly spreading the observed gold particles across all compartments according to their sizes. In practice, sizing is achieved by superimposing test probes (points and lines) on sections and counting chance encounters between the probes and cell compartments.
In $3 \mathrm{D}$ space, a membrane compartment presents as a surface and this appears on the cut plane of an ultrathin section as a linear trace or perimeter. Therefore, unlike sectional images of organelles, it cannot generate encounters with test points (the probability of a point hitting a line is zero). One solution to this situation is to convert the linear membrane trace into a profile sectional area. This socalled "acceptance zone" (Mayhew and Lucocq 2008) can be generated close to the membrane trace, the width of which corresponds approximately to the dispersion zone of gold label that occurs close to labelled membranes. Arbitrarily, this zone can be set according to the expected resolution (say, $20 \mathrm{~nm}$ for antibody followed by protein A gold) or by direct observations on the degree of dispersion. A useful rule of thumb is to adopt a zone with a distance from the membrane trace which is twice the diameter of the gold particles being used to label the membrane of interest. Often, gold particles are dispersed on both sides of the membrane irrespective of the location of the target molecules and, consequently, it may be sensible to adopt an acceptance zone on both sides of the membrane trace (in which case, overall width is equal to $2 w_{a}$ where $w_{a}$ is the distance from the membrane trace to one of the pair of zone boundaries).

The absolute profile area of the acceptance zone embracing both sides of the membrane trace can be found by multiplying its overall width by the profile length estimated by intersection counting. Alternatively, if RLI is to be used, the number of equivalent test points can be estimated for any a coherent stereological grid of test lines and points using $\left(c \times \Sigma I \times 2 w_{a}\right) / d$ where $\Sigma I$ is the sum of the intersections, $d$ is the test point spacing, $2 w_{a}$ is the overall width of the acceptance zone and $c$ is a constant for the coherent grid. For a square lattice grid in which the vertical and horizontal lines are separated by a spacing $\mathrm{d}$, the areal equivalent of a test point is $d^{2}$ and the grid constant is $c=$ $\pi / 4$. The total boundary trace length, $B$, of a given membrane trace is estimated as $B=c \times \Sigma I \times d$ and, for an overall acceptance zone width $2 w_{a}$; this gives an equivalent profile area of $c \times \Sigma I \times d \times 2 w_{a}$. However, this area is also equal to $\Sigma P \times d^{2}$ from which it follows that the number of equivalent test points $\Sigma P=\left(c \times \Sigma I \times 2 w_{a} \times d\right) / d^{2}$.

\section{Step 2: choose an appropriate approach for correcting membrane loss}

Often the majority of sectioned membrane traces belonging to one compartment are clear but a proportion may present indistinct images when the membrane is tilted relative to the section plane. To estimate the total expected gold particles it is necessary to correct for this image loss. The first step is to count gold associated with distinct membranes where the membrane is clearly visible and vertically 
sectioned (local vertical windows, LVWs). Correction factors are then applied to these counts and can be determined by estimating the fraction of poorly-imaged membrane. This can be performed in one of two ways: (1) goniometrically and (2) stereologically.

By means of goniometry, the critical angle to which a given membrane can be tilted in the section before it no longer appears as a clear trace can be determined directly. From these angles $(\theta)$, the fractional loss of membrane images $(F)$ can be calculated as

$F=1-([\sin 2 \theta+2 \theta] / \pi)$

and observed intersection counts on LVWs can be corrected accordingly from

$K=1 /(1-F)$

where $K$ is the correction factor.

The stereological approach is more limited in its applicability because it relies on using intersection counts to estimate the fraction of all membrane images which LVWs represent, $F=I_{\text {lost }} /\left(I_{\text {lost }}+I_{\mathrm{LVW}}\right)$. This approach works for the boundary membranes of compartments (e.g. nuclear envelope, plasma membrane) whose limits can be deduced even when the membrane images themselves are poorly defined or even unidentifiable. Unfortunately, the approach cannot be used directly on membranes whose images are not everywhere unambiguously identifiable (e.g. RER cisternae or mitochondrial cristae membranes). In such instances, it might be possible to use the fractional loss data from one group of membrane LVWs (e.g. those of the outer nuclear membrane) to correct the LVW counts for another membrane (e.g. RER cisternae).

Tilt-correction can be undertaken even when membranes that are tangentially sectioned cannot be seen at all. The gold and intersection counts are enlarged by the multiplier, $K$, and the intersection counts converted into point counts and inserted into the final dataset for statistical analysis. The statistical comparisons are performed as already described (see text and Tables 1 and 2).
Table 3 provides a synthetic dataset for a mixture of volume-occupying compartments (nucleoplasm, mitochondria, cytosol) and a surface occupier (RER membranes). The null hypothesis to be examined is that there is no preferential labelling over RER, i.e. no preferential association of the molecule of interest with this compartment. An overall acceptance zone of $2 w_{a}=0.04 \mu \mathrm{m}$ ( $w_{a}$ on each side of the membrane trace is $0.02 \mu \mathrm{m}$ ) was used and, by goniometry, the correction factor for lost membrane traces was estimated to be $K=9.3$ (Mayhew and Lucocq 2008). A total of 55 intersections were counted with clear (LVW) images of RER membranes and, multiplied by 9.3 , yielded a corrected figure of 511.5 intersections. Using a square test lattice with a line spacing of $d=0.5 \mu \mathrm{m}$, this corresponded to a test point count of $\Sigma P=(\pi / 4 \times 511.5 \times 0.04 \times 0.5) / 0.25=32.14$ falling on the RER acceptance zone. The observed gold particles on LVW images of RER (5) were also increased by 9.3 to obtain the corrected total of 46.5 .

Expected gold totals were calculated from the labelling density per test point multiplied by the compartmental point count. This was then compared with the observed gold count. For 3 degrees of freedom, the total $\chi^{2}$ value (63.73) indicates significance $(P<0.001)$ and the accompanying RLI value (2.78) and partial $\chi^{2}$ value $(83.2 \%$ of total) reveal that labelling is most concentrated on RER membranes.

Method 2: to compare the observed numbers of gold particles on compartments in different cell groups

In this case, the observed numerical frequency distributions of raw gold counts in different groups can be compared directly by contingency table analysis (Mayhew et al. 2002, 2004; Mayhew and Desoye 2004).

We illustrate how this is achieved using raw gold particle counts generated by a study of melanocyte-specific proteins in the lysosome-related disorder, HermanskyPudlak syndrome, HPS (Helip-Wooley et al. 2007). This study localized the melanosomal protein TYRP1 in human epidermal melanocytes from normal subjects and a patient

Table 3 Synthetic dataset to illustrate method 1 applied to an unspecified molecule in a mixture of volume- and surface-occupying compartments

\begin{tabular}{lllcllll}
\hline $\begin{array}{l}\text { Cellular } \\
\text { compartments }\end{array}$ & $\begin{array}{l}\text { Gold number, } \\
N_{\text {obs }}\end{array}$ & $\begin{array}{l}\text { Observed points } \\
\text { or intersections }\end{array}$ & $\begin{array}{l}\text { Corrected } \\
\text { points, } P\end{array}$ & $\begin{array}{l}\text { Expected } \\
\text { number, } N_{\text {exp }}\end{array}$ & LD = $N_{\text {obs }} / P$ & RLI $=\mathrm{LD}_{\text {comp }} / L_{\text {tot }}$ & $\chi^{2}$ values $(\%)$ \\
\hline Nucleoplasm & 31 & 60 & 60 & 31.21 & 0.517 & 0.99 & $0.00(0.0 \%)$ \\
RER membrane & $(5) 46.5$ & $(55) 511.5$ & 32.14 & 16.72 & 1.447 & 2.78 & $53.04(83.2 \%)$ \\
Cytosol & 57 & 150 & 150 & 78.02 & 0.380 & 0.73 & $5.66(8.9 \%)$ \\
Mitochondria & 6 & 28 & 28 & 14.56 & 0.214 & 0.41 & $5.03(7.9 \%)$ \\
Column totals & 140.5 & & 270.14 & 140.5 & 0.520 & 1.00 & $63.73(100 \%)$ \\
\hline
\end{tabular}

For total $\chi^{2}=63.73$ and 3 degrees of freedom (2-1 groups $\times 4-1$ compartments), $P<0.001$. The observed group distributions are significantly different and RLI and partial $\chi^{2}$ values indicate that RER is preferentially labelled (RLI $=2.78$ and partial $\chi^{2}$ accounts for $83 \%$ of total $\chi^{2}$ ) 
with HPS type 5. The dataset in Table 4 represents gold counts for TYRP1 detected using the monoclonal antibody, MEL5. The cells are compared in terms of six compartments: melanosomes, Golgi stacks + vesicles, extra-Golgi small vesicles, small tubules, early endosomes and late endosomes + multivesicular bodies + lysosomes.

For a given compartment in a given group, the number of expected gold particles is calculated by multiplying the corresponding column sum by the corresponding row sum and then dividing by the grand row sum. For example, the expected gold particles on the melanosome compartment in cells from normal patients is calculated as $53 \times 174 / 212=$ 43.50. With an observed gold count of 52, the partial $\chi^{2}$ amounts to $(52-43.50)^{2} / 43.50=1.66$ approximately.

The total $\chi^{2}$ value for the two groups of melanocytes is 47.26 and, for 5 degrees of freedom (2-1 groups $\times 6-1$ compartments), $P<0.001$. Therefore, the null hypothesis of no difference in distributions between groups is rejected. Inspection of partial $\chi^{2}$ values reveals that two compartments, the extra-Golgi vesicles and the melanosomes, are the principal contributors to these differences. In normal cells, there were fewer-than-expected gold particles on extra-Golgi small vesicles. In contrast, melanocytes from the HPS5 patient had more-than-expected particles on extra-Golgi vesicles but fewer-than-expected on melanosomes (Table 4).

Table 5 illustrates the method for the study referred to above on m-calpain distributions before and after 7 and 14 days of muscle conditioning (Borjigin et al. 2006). Again, the gold counts are based on observed LD values and our estimates of the fractional volume of sarcomere occupied by the A-band region. The total $\chi^{2}$ value for the three groups of muscle is 9.26 and, for 2 degrees of freedom (3-1 groups $-2-1$ compartments), $P<0.001$. The null hypothesis of no difference in distributions between groups must be rejected and examination of $\chi^{2}$ values shows that the major contributors to the differences resided in the controls and 14-day conditioned groups. The latter group had fewer-than-expected gold particles in the I-band/Z-disk and more-than-expected in the A-band. In contrast, the control muscle had more-than-expected particles in the Iband/Z-disk and fewer-than-expected in the A-band (Table 5).

For this between-group approach, magnification need not be known or standardized between groups. For statistical evaluation by contingency table analysis, it is advisable that expected numbers of gold particles should not be smaller than five and, again, this may influence the choice of compartments or numbers of sampled golds. It is also sensible to aim for similar column sums for total gold counts in each group of cells as statistical analysis may be distorted by large discrepancies between cell groups.

Since its introduction, the between-group method has been used to follow shifts in antigen distributions in different groups of cells (Mayhew et al. 2004; Mayhew and Desoye 2004; Santambrogio et al. 2005; Mühlfeld and Richter 2006; Nithipongvanitch et al. 2007). A potential disadvantage of the between-group comparison of observed gold counts is that it may limit mechanistic interpretation of shifts in labelling patterns. For example, a shift of receptor labelling from inside the cell and towards the cell membrane might be explained by an increase in the LD of the membrane (reflecting an increase in receptor concentration) or in the total amount of membrane (due to an increase in the surface area of cell membrane rather than a change in receptor concentration). In such cases, it may be better to supplement analysis by estimating LD or RLI values in order to compare compartments within each group (e.g. see Schmiedl et al. 2005).

The studies by Schmiedl et al. (2005) and Borjigin et al. (2006) serve further to illustrate that indications of preferential labelling in within-group comparisons do not

Table 4 Empirical dataset illustrating method 2

\begin{tabular}{|c|c|c|c|c|c|}
\hline Subcellular compartments & Normal & HP syndrome type 5 & Row totals & $\chi^{2}$ values & $\chi^{2}$ values as $\%$ \\
\hline Melanosomes & $52(43.50)$ & $1(9.50)$ & 53 & $1.66,7.61$ & $3.5,16.1$ \\
\hline Golgi stacks + vesicles & $14(11.49)$ & $0(2.51)$ & 14 & $0.55,2.51$ & $1.2,5.3$ \\
\hline Extra-Golgi vesicles & $30(45.96)$ & $26(10.04)$ & 56 & $5.54,25.38$ & $11.7,53.7$ \\
\hline Early endosomes & $21(18.88)$ & $2(4.12)$ & 23 & $0.24,1.09$ & $0.5,2.3$ \\
\hline Late endosomes, MVBs and lysosomes & $45(41.04)$ & $5(8.96)$ & 50 & $0.38,1.75$ & $0.8,3.7$ \\
\hline Small tubules & $12(13.13)$ & $4(2.87)$ & 16 & $0.10,0.45$ & $0.2,0.9$ \\
\hline Column totals & 174 & 38 & 212 & 47.26 & 100 \\
\hline
\end{tabular}

Localization of MEL5-identified TYRP1 in primary epidermal melanocytes from normal subjects and those with Hermansky-Pudlak syndrome type 5 (based on raw data in Helip-Wooley et al. 2007). Values represent observed (expected) numbers of 10 nm gold particles in each group. For total $\chi^{2}=47.26$ and 5 degrees of freedom ( $2-1$ groups $\times 6-1$ compartments), $P<0.001$. The observed group distributions are significantly different and partial $\chi^{2}$ values indicate that HP syndrome type 5 melanocytes have more-than-expected gold particles on small vesicles outside the Golgi apparatus and fewer-than-expected on melanosomes. In contrast, normal cells have fewer-than-expected particles on extra-Golgi small vesicles 
Table 5 Empirical dataset illustrating method 2

\begin{tabular}{lllllll}
\hline Sarcomere regions & Controls & 7-day conditioned & 14-day conditioned & Row totals & $\chi^{2}$ values & $\chi^{2}$ values as $\%$ \\
\hline I-band/Z-disk & $264(243.5)$ & $664(641.5)$ & $616(659.0)$ & 1,544 & $1.73,0.79,2.80$ & $18.6,8.5,30.3$ \\
A-band & $308(328.5)$ & $843(865.5)$ & $932(889.0)$ & 2,083 & $1.28,0.58,2.08$ & $13.8,6.3,22.4$ \\
Column totals & 572 & 1,507 & 1,548 & 3,627 & 9.26 & 100 \\
\hline
\end{tabular}

Localization of $\mathrm{m}$-calpain in two sarcomere regions of bovine serratus ventralis muscle before and after post-mortem conditioning by storage at $2-4^{\circ} \mathrm{C}$ (see text and Borjigin et al. 2006). Values represent observed (expected) numbers of $10 \mathrm{~nm}$ gold particles in each group. For total $\chi^{2}=$ 9.26 and 2 degrees of freedom, $P<0.01$. The observed group distributions are significantly different and the controls and 14-day conditioned groups account for the differences. Controls had more-than-expected gold particles in the I-band/Z-disk regions and fewer-than-expected in the A-band whereas the 14-day conditioned muscle had fewer-than-expected gold particles in the former, and more-than-expected, in the latter regions

necessarily imply the same distribution pattern in betweengroup comparisons. In the case of m-calpain distributions (Borjigin et al. 2006), there were significant differences between control and 14-day conditioned groups (Table 5) despite the detection of preferential labelling of I-band/Zdisk regions of sarcomeres in both groups (Tables 1 and 2). In the same way, Schmiedl et al. (2005) found preferential labelling of lamellar bodies and multivesicular bodies for surfactant protein B (SP-B) in pneumocytes from newborn, 14-day old, and adult rat lungs. However, raw data taken from their study reveal that there are still distributional shifts of labelling between these groups (Table 6).

\section{Labelling efficiency and its estimation}

Gold particle labelling is a readout of underlying molecular components located in a thin section. However, in postembedding (or on-section) labelling, not all target molecules become labelled by gold particles and, sometimes, more than one gold particle might be associated with a given target. To take account of these effects, the concept of labelling efficiency (LE) has been introduced. LE is equal to the number of golds per antigen molecule, $N_{g} / N_{m}$, and can be expressed as a decimal fraction or a percentage.
Thus, an LE value of 0.10 (or $10 \%$ ) reports that, on average, each gold particle is associated with ten target molecules. The quantity cannot be identified without resort to a pilot investigation because it is influenced by many factors including fixation conditions, embedding medium, immunolabelling reagents and labelling protocols. Importantly, even when preparation conditions are constant, LE varies between compartments mainly because of differential penetration of labelling reagents into the section. Methods for estimating LE require some form of calibration which may involve (1) producing standard reference gels with known amounts of antigen or (2) making tandem biochemical estimates of the amounts of the target molecules.

\section{Reference gels}

The idea here is to introduce a known concentration of purified target molecules (e.g. protein of interest) into a gel that is processed and sectioned along with the cell sample. Gels and sample are sectioned and then labelled under identical conditions and gold signals compared. This is achieved by assessing LD over different regions of the calibration gel that possess a range of antigen

Table 6 Empirical dataset illustrating method 2

\begin{tabular}{lllllll}
\hline Cell compartments & Newborn & 14-day old & Adult & Row totals & $\chi^{2}$ Values & $\chi^{2}$ values as $\%$ \\
\hline Lamellar bodies & $330(465.53)$ & $687(583.65)$ & $592(559.82)$ & 1,609 & $39.46,18.30,1.85$ & $3.5,16.1$ \\
Mitochondria & $74(83.62)$ & $93(104.83)$ & $122(100.55)$ & 289 & $1.11,1.34,4.57$ & $1.2,5.3$ \\
Cytoplasm & $663(586.76)$ & $694(735.63)$ & $671(705.60)$ & 2,028 & $9.91,2.36,1.70$ & $11.7,53.7$ \\
Multivesicular bodies & $44(41.95)$ & $44(52.60)$ & $57(50.45)$ & 145 & $0.10,1.41,0.85$ & $0.5,2.3$ \\
Nucleus & $706(639.13)$ & $760(801.29)$ & $743(768.58)$ & 2,209 & $7.00,2.13,0.85$ & $0.8,3.7$ \\
Column totals & 1817 & 2,278 & 2,185 & 6,280 & 92.92 & 100
\end{tabular}

Localization of surfactant protein B (SP-B) in type II pneumocytes from newborn, 14-day old and adult rats (based on raw data in Schmiedl et al. 2005). Values represent observed (expected) numbers of 5 or $10 \mathrm{~nm}$ gold particles in each group. For total $\chi^{2}=92.92$ and 8 degrees of freedom, $P$ $<0.001$. The observed group distributions are significantly different and partial $\chi^{2}$ values indicate that cells in newborn have less-than-expected gold particles on lamellar bodies and more-than-expected in cytoplasm. In 14-day old rats, there are more-than-expected particles on lamellar bodies. RLI values within each age group (Schmiedl et al. 2005) revealed that distributions were non-random at all ages with lamellar bodies and multivesicular bodies being preferentially labelled. The above analysis reveals that the patterns of preferential labelling also vary between groups 
concentrations, thereby generating a calibration curve. The LD over compartments of interest is then estimated and the antigen concentration read from the calibration curve. Variants of this approach have been used to estimate antigen concentration in the secretory pathway and for amino acid neurotransmitters in compartments of neuronal cells (Ottersen and Storm-Mathisen 1984; Ottersen 1987a, b; Slot et al. 1989; Posthuma et al. 1987, 1988). Such studies showed that the embedding matrix can act to equalise LE in different compartments by limiting penetration of the labelling regents into the section. This approach has some inherent assumptions including: (a) that the gel undergoes equal dimensional changes during the fixation, embedding, sectioning and labelling processes and (b) that LE in the gel is the same as in the cell compartment of interest.

Biochemical measurements

\section{Comparing labelling density with biochemical assay of antigen membrane density}

In a series of pioneering studies (Griffiths et al. 1983; Quinn et al. 1984; Griffiths and Hoppeler 1986), the aim was to estimate the LE of Semliki Forest virus spike glycoproteins in the secretory pathway. There were two main elements to the estimates. First, the number of virus membrane glycoprotein molecules $\left(N_{m}\right)$ in different membrane compartments of the average infected cell was determined by a combination of biochemistry and stereological estimation of the total membrane surface of a compartment $\left(S_{c}\right)$. These values were then combined to derive estimates of the molecular density $\left(N_{m} / S_{c}\right)$ in ER, Golgi and plasma membrane (Griffiths et al. 1983; Quinn et al. 1984). Second, immunoelectron microscopy (Griffiths and Hoppeler 1986) was used to label the same glycoprotein in ultrathin sections of infected cells and obtain an LD over membranes, expressed as the number of gold particles per $\mu \mathrm{m}^{2}$ of membrane surface $\left(N_{g} / S_{c}\right)$. The gold density was then related to the estimates of antigen concentration in each compartment to give the LE as follows:

$\mathrm{LE}=\left(N_{g} / S_{c}\right) /\left(N_{m} / S_{c}\right)=N_{g} / N_{m}$.

The following steps describe how estimates of the gold labelling densities were obtained. Stereological estimation of the LD of a membrane compartment requires that encounters between test probes (lines) and surfaces are isotropic and so sectioning must be IUR or test lines must be IUR.

Step 1. The number of gold particles per organelle profile area (e.g. the planar area of ER cisternae) was estimated by test point counting:
$N_{g} / A_{c}=N_{g} / \sum P_{c} a_{p}$

where $\Sigma P_{c}$ represents the test point total summed over all randomly-sampled fields and $a_{p}$ is the test lattice constant (i.e. the area associated with a test point given on the specimen scale by correcting for the areal magnification). The number of golds was related to the volume of organelle in the section by dividing the number per unit area by section thickness, $t$ :

$N_{g} / V_{c}=\left(N_{g} / A_{c}\right) / t$.

Step 2. The membrane surface density of the compartment (expressed as the surface area per unit volume of the compartment, $\left.S_{c} / V_{c}\right)$ was estimated by intersection counting using IUR sections:

$S_{c} / V_{c}=2 \sum I_{c} / \sum P_{c} l_{p}$.

Here, $\Sigma I_{c}$ represents the number of test line intersections with a membrane compartment (e.g. RER membrane) summed over all fields, $\Sigma P_{c}$ is the organelle point total (e.g. RER cisternal lumen) and $l_{p}$ is the lattice constant (i.e. the test line length associated with a test point given on the specimen scale by taking into account the linear magnification).

Step 3. The number of gold particles per unit of membrane surface was calculated by dividing the particle density per unit volume by the membrane surface density:

$N_{g} / S_{c}=\left(N_{g} / V_{c}\right) /\left(S_{c} / V_{c}\right)$.

Using cryosections, the resulting estimates of LE for viral spike proteins were 0.40 for ER and 0.13 for the Golgi complex (Griffiths and Hoppeler 1986). In other words, the number of gold particles counted over ER and Golgi represented $40 \%$ and $13 \%$, respectively, of the total membrane viral spike glycoprotein molecules in the cell. Efficiencies were found to be lower in Lowicryl sections ( 0.18 and 0.07 , respectively, for ER and Golgi).

\section{Comparing labelling with biochemical assay of antigen per cell}

Lucocq (1992) presented a method of quantifying the LE of a cell surface-located enzyme (horseradish peroxidase, HRP) in cells embedded in Lowicryl resins at low temperature. The method combined biochemical measurement of the number of enzyme molecules with stereological estimates of the amount of gold label associated with the plasma membrane, both quantities being expressed on a per cell basis. The stereological method used the physical disector, a volume probe comprising a pair of sections separated by a known distance, $h$ (Sterio 1984; Gundersen 1986). In fact, a double disector (Gundersen 1986; Lucocq 
1992, 1994) from a single stack of sections was used to estimate both the packing density of cells in a reference volume $\left(N_{\text {cell }} / V_{\text {ref }}\right.$, where ref represents the centrifuge pellet) and the packing density of gold particles in the same reference volume $\left(N_{g} / V_{\text {ref }}\right)$. The number of gold particles in the average cell is then given by the relationship:

$N_{g} / N_{\text {cell }}=\left(N_{g} / V_{\text {ref }}\right) /\left(N_{\text {cell }} / V_{\text {ref }}\right)$.

Stereological estimation of packing densities in a volume requires that encounters between test probes (disectors) and cells are random in position. The procedure adopted was as follows:

Step 1. A stack of serial thin sections was prepared and, using unbiased forbidden line counting frames (Gundersen 1977; Sterio 1984) containing a total of $\Sigma P_{\text {tot } 1}$ test points, the number of cells per reference volume $\left(N_{\text {cell }} / V_{\text {ref }}\right)$ was estimated by counting cells which disappeared from one section plane to the other, $\Sigma Q_{\text {cell }}{ }^{-}$. With a counting frame area, $a_{1}$, and $\Sigma P_{\text {cell1 }}$ points hitting the cells in the pellet, the numerical density of cells over a randomly-sampled set of disectors, separated by a known spacing $h_{1}$, was estimated as follows:

$N_{\text {cell }} / V_{\text {ref }}=\sum Q_{\text {cell }}^{-} \cdot \sum P_{\text {tot } 1} / 2 \sum P_{\text {cell1 }} a_{1} h_{1}$

where the factor 2 is included because cells are counted in both directions with physical disectors and $a_{1}$ denotes the frame area given on the specimen scale by taking into account the areal magnification.

Step 2. A single section was randomly selected from the original stack and labelled for the antigen. An SUR sample of fields was selected and an SUR sample of rectangular counting frames of area $a_{2}$, and containing a total of $\Sigma P_{\text {tot } 2}$ test points, was superimposed. Within these counting frames, the number of gold particles associated with plasma membranes, $N_{g}$, and the number of test points hitting cell profiles, $\Sigma P_{\text {cell2, }}$ were counted. The number of gold particles per cell profile area was estimated as $N_{g} \Sigma P_{\text {tot } 2} / \Sigma P_{\text {cell2 }} a_{2}$. An estimate of the number per unit volume of cell was given by multiplying total cell area by the disector height $\left(h_{2}\right)$ which, in the original study, was equal to section thickness.

Step 3. The number of golds associated with the average cell was calculated from the two numerical densities. Since all sections in the stack were cut at the same thickness, the ratio $h_{1} / h_{2}$ simply represents the number of sections, $\mathrm{n}$, in the original stack and the final equation becomes:

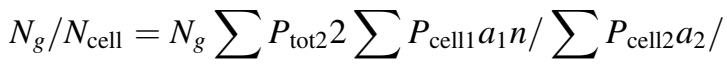

$$
\begin{aligned}
& \sum Q_{\text {cell }}^{-} \sum P_{\text {tot1 }} \text {. }
\end{aligned}
$$

Using Lowicryl resin sections, an LE of about 0.03 was obtained when stereological estimates were referred to biochemical determination of the numbers of HRP molecules per average cell (Lucocq 1994).
Additional developments

A stereological method for estimating the numbers of immunogold-labelled antigens in cells within tubules has been developed recently (Razga and Nyengaard 2006) and this, too, could be used to calculate LE values for such cells. However, its primary purpose has been to estimate the number of angiotensin II AT1 receptors of vascular endothelial and smooth muscle cells in the afferent and efferent arterioles of rat kidney (Razga and Nyengaard 2007). Based on $10-\mu \mathrm{m}$-thick frozen sections, and on semithin and ultrathin sections prepared from them, the method involves pre-embedding labelling of AT1 receptors using ultrasmall gold particles (diameter ca. $0.8 \mathrm{~nm}$ ), which gives good penetration throughout the tissue rather than just at the section surface. This was followed by silver enhancement which increased effective particle size to ca. $35 \mathrm{~nm}$. Arterioles were sampled using disectors (Sterio 1984) and their length between section planes estimated from axial ratios and section thickness. The volume of cells within the same section was estimated by point counting using the Cavalieri principle (Gundersen and Jensen 1987) and the numerical density of enhanced gold particles in cell volume was estimated on 70-nm-thick resin sections. From these intermediate steps, it was possible to estimate the number of particles per arteriole and per arteriolar cell complement (Razga and Nyengaard 2006). Using these methods, it was found that the number of immunogold-labelled receptors is greater in renin-negative, compared to renin-positive, smooth muscle cells of arterioles and that endothelial and smooth muscle cells are similar in the relative numbers of receptors (Razga and Nyengaard 2007).

Spatial patterns of gold labelling within particular compartments, notably cell membranes, have been studied in other ways. For example, computer simulation using different models (e.g. membrane rafts) has been applied in an attempt to reproduce the arrangement of gold particles on an actual membrane surface from the observed distribution of particles on sectional traces of membrane (Nikonenko et al. 2000). This approach could prove useful when spatial arrangements of gold particles cannot be viewed directly, e.g. by scanning electron microscopy, goniometry or use of membrane sheets (Prior et al. 2003; Meredith et al. 2004; Wilson et al. 2004; Socher and Benayahu 2008). At present, application has been limited to membranes with extremely low surface curvature (Nikonenko et al. 2000). Recently, conventional and environmental scanning electron microscopy have been used in conjunction with immunogold labelling, with or without silver enhancement, to detect proteins at cell surfaces (Socher and Benayahu 2008). Rigorous methods for quantifying the resulting images, taking account of surface curvature effects, are awaited. 
An alternative approach examines the clustering of gold particles to define the location of compartments which cannot be identified on morphological criteria alone (Schöfer et al. 2004). In combination with TEM in situ hybridization, this approach has been used to detect individual chromosomal domains in the nuclei of HeLa cells. Because the ability to distinguish labelled compartments depends on differences in LD between them and their milieu, the thresholding process works optimally when background labelling is low and the compartment-milieu interface is smooth rather than irregular in outline. It remains to be seen how useful this method would be if it was required to resolve compartments sharing similar LD values.

A further possible future development is the combination of stereology and electron tomography (Vanhecke et al. 2007). With electron tomography, stacks of parallel "optical" slices can be generated from thick (200-400 nm) TEM sections with high resolution and these slices can be used in combination with stereological sampling and estimation tools to estimate relevant structural quantities such as volume, surface area and number. Crucially, with tomography, section thickness can be reduced to a few nanometres and this makes the technique well suited to stereological analysis of small structures which, in thicker $(50-100 \mathrm{~nm})$ sections are liable to biases which are not easy to correct. In combination with pre-embedding immunogold labelling and section penetration, the techniques could be used to determine absolute numbers of particles as well as LD values expressed as number per $\mu \mathrm{m}^{3}$ of compartment volume or per $\mu \mathrm{m}^{2}$ of compartment surface.

Though more suited to dealing with individual cells, rather than aggregate subcellular compartments, Wessendorf et al. (2004) have described the use of contingency testing with $\chi^{2}$ or Fisher's exact tests to detect preferential labelling. This method has been applied to cell preparations labelled autoradiographically by in situ hybridization, but is potentially applicable to those labelled with colloidal gold. Essentially, this method compares the density of labelling of a cell with that of its surroundings and so represents a more focused variant of the contingency testing described herein. However, it could be adapted to test for preferential labelling of an aggregate (e.g. a group of cells) in comparison to the surroundings.

Finally, areas of considerable interest at subcellular (and higher) levels of organisation are colocalization of different antigens and quantification of nanoparticles in general. Correlation function analysis has been used to quantify colocalization patterns of nascent DNA with different nuclear proteins in HeLa cells and lipid raft markers in mast tumour cell membranes (Philimonenko et al. 2000; Wilson et al. 2004). It is worthy of note that the LD and RLI methods described above could also be applied to assess colocalization patterns. For instance, if different sizes of gold particle were used to label two antigens, evidence for colocalization could be shown if the labelling distributions between compartments, or within a compartment, was the same with both sizes of particle. Extensions of current methods have also been applied to count nanoparticles at cell and organ levels (Mühlfeld et al. 2007a, b).

Comments and concluding remarks

\section{General comments}

Accurate quantitative localisation of cell components is increasingly important in cell biological and signalling studies. For this purpose, immunoelectron microscopy has a number of distinct advantages over light-based methods. First, it provides a resolution approximately tenfold higher than conventional optical microscopy (20 vs. $200 \mathrm{~nm}$ ). Second, it provides unrivalled amounts of morphological and spatial information by visualising the "structural context" onto which labelling quantities are mapped. Third, it allows rigorous fixation methods and therefore excellent structural preservation. Last, quantitative approaches such as the new methods described here, provide the precision, sensitivity and unbiasedness that are prerequisites of sound quantitative localisation of cell components. These methods provide a statistically robust and rapid format for quantitative comparisons over multiple compartments. Also, because they employ a digital gold signal that can be unambiguously identified, all the signal over a compartment can be collected even if it is low and widely dispersed.

\section{The role of the new methods in labelling studies}

So how might the new methods contribute to a cell biological study? One possible contribution would be in the initial stages of a labelling study when new antibodies are being tried at different concentrations with the aim of identifying candidate compartments for specific labelling. At higher concentrations of antibody, preferential labelling of a compartment by non-specific interactions is more likely and may occur alongside specific binding. However, as the antibody is diluted, preferential labelling due to nonspecific interactions will be reduced, while the true specific labelling of the target molecule should become predominant because of the high affinity/avidity of the antibodies. The emergence of specifically-labelled compartments will then be revealed as quantitative or qualitative changes in preferentially-labelled compartments or in the distribution of labelling.

Once candidate compartment for specific labelling has been identified using a dilution series, specificity controls 
may be carried out. The best controls aim to change the amount or concentration of the target molecule in situ. Examples would be knockdown of protein expression by gene deletion or by the use of small interfering RNA. The resulting distributions of label could be compared using the techniques described here (method 2). Once specific label has been identified, its extent can be assessed by removing the residual label present after knockdown of protein. This can be performed by subtracting the labelling density of knockdown from the control over each compartment. The residual $\mathrm{LD}$ can then be combined with the point or intersection counts to recalculate the distribution which could then be assessed for preferential distribution by adopting method 1 (for details, see Watt et al. 2004). Another possible use for these new methods would be to compare gold labelling under different experimental conditions when specificity of labelling is known. Here, changes in the quantity/distribution of a target component, consequent on differences between biology-based experiments, may be detected by these methods. Though a number of examples have been described, it should be noted that these methods concentrate on the pattern of gold labelling across compartments and significance is tested using the non-parametric $\chi^{2}$ analysis which is sensitive to differences in distributions rather than comparing individual compartments. Also, the methods do not address the issue of reproducibility of the observations or address the key question of how much more or less labelling there is over any individual compartment in different experimental conditions. In the future, the challenge will be to develop these methods further to combine statistical assessment of distributions with measures of reproducibility and extent in the analysis of digital gold signal. As to the former, a possible way forward is simply to assess reproducibility by replication, i.e. by analysing at least two paired or unpaired sets of data from each of the control and experimental groups.

Acknowledgments We are grateful to many colleagues with whom we have collaborated and freely exchanged ideas over the years. We also thank Jürgen Roth and the Editorial Team of Histochemistry and Cell Biology for providing an excellent vehicle for promoting the new gold-counting methods over the last 5 years.

\section{References}

Baddeley AJ, Gundersen HJG, Cruz-Orive LM (1986) Estimation of surface area from vertical sections. J Microsc 142:259-276

Bendayan M (2001) Worth its weight in gold. Science 291:1363-1365

Bennett PM, Baines AJ, LeComte M-C, Maggs AM, Pinder JC (2004) Not just a plasma membrane protein: in cardiac muscle cells alpha-II spectrin also shows a close association with myofibrils. J Muscle Res Cell Motil 25:119-126

Borjigin G, Yamamoto S, Sugiyama T, Nishiumi T, Suzuki A (2006) Changes in the immunogold electron-microscopic localization of calpain in bovine skeletal muscle induced by conditioning and high-pressure treatment. Biosci Biotechnol Biochem 70:12491254

Cernadas M, Sugita M, van der Wel N, Cao XC, Gumperz JE, Maltsev S, Besra GS, Behar SM, Peters PJ, Brenner MB (2003) Lysosomal localization of murine CD1d mediated by AP-3 is necessary for NK T cell development. J Immunol 171:41494155

Coene ED, Hollinshead MS, Waeytens AAT, Schelfhout VRJ, Eechaute WP, Shaw MK, Van Oostveldt PMV, Vaux DJ (2005) Phosphorylated BRCA1 is predominantly located in the nucleus and mitochondria. Mol Biol Cell 16:997-1010

Davey KAB, Garlick PB, Warley A, Southworth R (2007) Immunogold labeling study of the distribution of GLUT-1 and GLUT-4 in cardiac tissue following stimulation by insulin or ischemia. Am J Physiol Heart Circ Physiol 292:H2009-H2019

Driskell OJ, Mironov A, Allan VJ, Woodman PG (2007) Dynein is required for receptor sorting and the morphogenesis of early endosomes. Nature Cell Biol 9:113-120

Fehrenbach H, Tews S, Fehrenbach A, Ochs M, Wittwer T, Wahlers $\mathrm{T}$, Richter J (2005) Improved lung preservation relates to an increase in tubular myelin-associated surfactant protein A. Respir Res 6:1-12

Fujii J, Matsui T, Heatherly DP, Schlegel KH, Lobo PI, Yutsudo T, Ciraolo GM, Morris RE, Obrig T (2003) Rapid apoptosis induced by Shiga toxin in HeLa cells. Infect Immunol 71:2724-2735

Griffiths G (1993) Fine structure immunocytochemistry. Springer, Heidelberg

Griffiths G, Hoppeler H (1986) Quantitation in immunocytochemistry: correlation of immunogold labelling to absolute number of membrane antigens. J Histochem Cytochem 34:1389-1398

Griffiths G, Warren G, Quinn P, Mathieu-Costello O, Hoppeler H (1983) Density of newly synthesized plasma membrane proteins in intracellular membranes. I. Stereological studies. J Cell Biol 98:2133-2141

Griffiths G, Lucocq JM, Mayhew TM (2001) Electron microscopy applications for quantitative cellular microbiology. Cell Microbiol 3:659-668

Gundersen HJG (1977) Notes on the estimation of the numerical density of arbitrary profiles: the edge effect. J Microsc 111:219223

Gundersen HJG (1986) Stereology of arbitrary particles. A review of unbiased number and size estimators and the presentation of some new ones, in memory of William R. Thompson. J Microsc 143:3-45

Gundersen HJG, Jensen EB (1987) The efficiency of systematic sampling in stereology and its prediction. J Microsc 147:229_ 263

Gundersen HJG, Jensen EBV, Kiêu K, Nielsen J (1999) The efficiency of systematic sampling in stereology-reconsidered. J Microsc 193:199-211

Helip-Wooley A, Westbroek W, Dorward HM, Koshoffer A, Huizing M, Boissy RE, Gahl WA (2007) Improper trafficking of melanocyte-specific proteins in Hermansky-Pudlak syndrome type-5. J Invest Dermatol 127:1471-1478

Hundorfean G, Zimmer K-P, Strobel S, Gebert A, Ludwig D, Büning $G$ (2007) Luminal antigens access late endosomes of intestinal epithelial cells enriched in MHC I and MHC II molecules: in vivo study in Crohn's ileitis. Am J Physiol Gastrointest Liver Physiol 293:G798-G808

Jacob M, Mannherz HG, Napirei M (2007) Chromatin breakdown by deoxyribonuclease 1 promotes acetaminophen-induced liver necrosis: an ultrastructural and histochemical study on male CD-1 mice. Histochem Cell Biol 128:19-33

Kweon HS, Beznoussenko GV, Micaroni M, Polishchuk RS, Trucco A, Martella O, Di Giandomenico D, Marra P, Fusella A, Di 
Pentima A, Berger EG, Geerts WJC, Koster AJ, Burger KNJ, Luini A, Mironov AA (2004) Golgi enzymes are enriched in perforated zones of Golgi cisternae but are depleted in COPI vesicles. Mol Biol Cell 15:4710-4724

Li ACY, Warley A, Thoree V, Simpson RJ, Mckie AT, Kodjabashia K, Thompson RPH, Powell JJ (2006) Immunolocalization of duodenal cytochrome B: a relationship with circulating markers of iron status. Eur J Clin Invest 36:890-898

Lopes MS, Cortadellas N, Kichey T, Dubois F, Habash DZ, Araus JL (2006) Wheat nitrogen metabolism during grain filling: comparative roles of glumes and the flag leaf. Planta 225:165-181

Lucocq JM (1992) Quantitation of gold labelling and estimation of labelling efficiency with a stereological counting method. J Histochem Cytochem 40:1929-1936

Lucocq JM (1993) Unbiased 3-D quantitation of ultrastructure in cell biology. Trends Cell Biol 3:354-358

Lucocq JM (1994) Quantitation of gold labelling and antigens in immunolabelled ultrathin sections. J Anat 184:1-13

Lucocq JM (2003) Electron microscopy in cell biology. In: Davey J, Lord M (eds) Essential Cell Biology. A Practical Approach, Oxford University Press, pp 53-112

Lucocq JM (2006) Quantitative EM techniques. In: Jorde LB, Jorde LB, Little PFR, Dunn MJ, Subramaniam S (eds) Encyclopedia of genetics, genomics, proteomics and bioinformatics. Wiley, Chichester. doi:10.1002/047001153X.g304217

Lucocq JM (2008) Quantification of structures and gold labelling for transmission electron microscopy. In: Allen T (eds) Methods in cell biology, American Society for Cell Biology and Academic Press (in press)

Lucocq JM, Habermann A, Watt S, Backer J, Mayhew TM, Griffiths G (2004) A rapid method for assessing the distribution of gold labelling on thin sections. J Histochem Cytochem 52:991-1000

Mattfeldt T, Mall G, Gharehbaghi H, Möller P (1990) Estimation of surface area and length with the orientator. J Microsc 159:301317

Maunsbach AB, Afzelius BA (1999) Biomedical electron microscopy. Illustrated methods and interpretations. Academic Press, San Diego

Mayhew TM (1991) The new stereological methods for interpreting functional morphology from slices of cells and organs. Exp Physiol 76:639-665

Mayhew TM (2007a) Quantitative immunoelectron microscopy. Alternative ways of assessing subcellular patterns of gold labelling. In: Kuo J (ed) Methods in molecular biology, vol 369, electron microscopy: methods and protocols, 2nd edn. Humana Press Inc, Totowa, pp 309-329

Mayhew TM (2007b) Counting your gold: comparing the relative labelling indices of cellular compartments in a mixture of organelles and membranes. J Anat 209:634-635

Mayhew TM (2008) Taking tissue samples from the placenta: an illustration of principles and strategies. Placenta 29:1-14

Mayhew TM, Desoye G (2004) A simple method for comparing immunogold distributions in two or more experimental groups illustrated using GLUT1 labelling of isolated trophoblast cells. Placenta 25:580-584

Mayhew TM, Lucocq JM (2008) Quantifying immunogold labelling patterns of cellular compartments when they comprise mixtures of membranes (surface-occupying) and organelles (volumeoccupying). Histochem Cell Biol 129:367-378

Mayhew TM, Lucocq JM, Griffiths G (2002) Relative labelling index: a novel stereological approach to test for non-random immunogold labelling of organelles and membranes on transmission electron microscopy thin sections. J Microsc 205:153-164

Mayhew T, Griffiths G, Habermann A, Lucocq J, Emre N, Webster P (2003) A simpler way of comparing the labelling densities of cellular compartments illustrated using data from VPARP and
LAMP-1 immunogold labelling experiments. Histochem Cell Biol 119:333-341

Mayhew TM, Griffiths G, Lucocq J (2004) Applications of an efficient method for comparing immunogold labelling patterns in the same sets of compartments in different groups of cells. Histochem Cell Biol 122:171-177

Mazzone M, Baldassarre M, Beznoussenko G, Giacchetti G, Vao J, Zucker S, Luini A, Buccione R (2004) Intracellular processing and activation of membrane type 1 matrix metalloprotease depends on its partitioning into lipid domains. J Cell Sci 117:6275-6287

Meredith DO, Owen GR, Ap Gwynn I, Richards RG (2004) Variation in cell-substratum adhesion in relation to cell cycle phases. Exp Cell Res 293:58-67

Mironov A, Latawiec D, Wille H, Bouzamondo-Bernstein E, Legname G, Williamson RA, Burton D, DeArmond SJ, Prusiner SB, Peters PJ (2003) Cytosolic prion protein in neurons. J Neurosci 23:7183-7193

Mühlfeld C, Richter J (2006) High-pressure freezing and freeze substitution of rat myocardium for immunogold labelling of connexin 43. Anat Rec 288A:1059-1067

Mühlfeld C, Mayhew TM, Gehr P, Rothen-Rutishauser B (2007a) A novel quantitative method for analysing the distributions of nanoparticles between different tissue and intracellular compartments. J Aerosol Med 20:395-407

Mühlfeld C, Geiser M, Kapp N, Gehr P, Rothen-Rutishauser B (2007b) Re-evaluation of pulmonary titanium dioxide nanoparticle distribution using the "relative deposition index": evidence for clearance through microvasculature. Part Fibre Toxicol 4:7 (8 pages)

Nikonenko AG, Nikonenko IR, Skibo GG (2000) Computer simulation approach to the quantification of immunogold labelling on plasma membrane of cultured neurons. J Neurosci Methods 96:11-17

Nithipongvanitch R, Ittarat W, Velez JM, Zhao R, St Clair DK, Oberley TD (2007) Evidence for p53 as guardian of the cardiomyocyte mitochondrial genome following acute adriamycin treatment. J Histochem Cytochem 55:629-639

Nyengaard JR, Gundersen HJG (1992) The isector: a simple and direct method for generating isotropic, uniform random sections from small specimens. J Microsc 165:427-431

Nyengaard JR, Gundersen HJG (2006) Direct and efficient stereological estimation of total cell quantities using electron microscopy. J Microsc 222:182-187

Ochs M (2006) A brief update on lung stereology. J Microsc 222:188-200

Ochs M, Johnen G, Müller K-M, Wahlers T, Hawgood S, Richter J, Brasch F (2002) Intracellular and intraalveolar localization of surfactant protein A (SP-A) in the parenchymal region of the human lung. Am J Respir Cell Mol Biol 26:91-98

Ottersen OP (1987a) Postembedding light and electron microscopic immunocytochemistry of amino acids: description of a new model system allowing identical conditions for specificity testing and tissue processing. Exp Brain Res 69:167-174

Ottersen OP (1987b) Postembedding immunogold labelling of fixed glutamate: an electron microscopic analysis of the relationship between gold particle density and antigen concentration. J Chem Neuroanat 2:57-66

Ottersen OP, Storm-Mathisen J (1984) Glutamate- and GABAcontaining neurons in the mouse and rat brain, as demonstrated with a new immunocytochemical technique. J Comp Neurol 229:374-392

Philimonenko AA, Janáček J, Hozák P (2000) Statistical evaluation of colocalization patterns in immunogold labelling experiments. $\mathbf{J}$ Struct Biol 132:201-210

Posthuma G, Slot JW, Geuze HJ (1987) Usefulness of the immunogold technique in quantitation of a soluble protein in ultra-thin sections. J Histochem Cytochem 35:405-410 
Posthuma G, Slot JW, Veenendaal T, Geuze HJ (1988) Immunogold determination of amylase concentrations in pancreatic subcellular compartments. Eur J Cell Biol 46:327-335

Potolicchio I, Chitta S, Xu X, Fonseca D, Crisi G, Horejsi V, Strominger JL, Stern LJ, Raposo G, Santambrogio L (2005) Conformational variation of surface class II MHC proteins during myeloid dendritic cell differentiation accompanies structural changes in lysosomal MIIC. J Immunol 175:4935-4947

Prior IA, Muncke C, Parton RG, Hancock JF (2003) Direct visualization of Ras proteins in spatially distinct cell surface microdomains. J Cell Biol 160:165-170

Quinn P, Griffiths G, Warren G (1984) Density of newly synthesized plasma membrane proteins in intracellular membranes. II. Biochemical studies. J Cell Biol 98:2142-2147

Razga Z, Nyengaard JR (2006) A stereologic approach to estimate the number of immunogold-labeled molecules in cells of tubules. Anal Quant Cytol Histol 28:54-60

Razga Z, Nyengaard JR (2007) Estimation of the number of angiotensin II AT1 receptors in rat kidney afferent and efferent arterioles. Anal Quant Cytol Histol 29:208-216

Santambrogio L, Potolicchio I, Fessler SP, Wong S-H, Raposo G, Strominger JL (2005) Involvement of caspase-cleaved and intact adaptor protein 1 complex in endosomal remodelling in maturing dendritic cells. Nature Immunol 6:1020-1028

Schmiedl A, Ochs M, Mühlfeld C, Johnen G, Brasch F (2005) Distribution of surfactant proteins in type II pneumocytes of newborn, 14-day old, and adult rats: an immunoelectron microscopic and stereological study. Histochem Cell Biol 124:465-476

Schöfer C, Janáček J, Weipoltshammer K, Pourani J, Hozák P (2004) Mapping of cellular compartments based on ultrastructural immunogold labelling. J Struct Biol 147:128-135

Signoret N, Hewlett L, Wavre S, Pelchen-Matthews A, Oppermann M, Marsh M (2005) Agonist-induced endocytosis of CC chemokine receptor 5 is clathrin dependent. Mol Biol Cell 16:902-917

Skepper JN (2000) Immunocytochemical strategies for electron microscopy: choice or compromise. J Microsc 199:1-36

Slot JW, Posthuma G, Chang LY, Crapo JD, Geuze HJ (1989) Quantitative aspects of immunogold labelling in embedded and in nonembedded sections. Am J Anat 46:327-335

Slot JW, Geuze HJ, Gigengack S, James DE, Lienhard GE (1991) Translocation of the glucose transporter GLUT4 in cardiac myocytes of the rat. Proc Natl Acad Sci USA 88:7815-7819

Socher R, Benayahu D (2008) Comparative study using scanning electron techniques for imaging of micro-architecture and antigen appearance. J Microsc 230:233-239

Sosinsky GE, Giepmans BN, Deerinck TJ, Gaietta GM, Ellisman MH (2007) Markers for correlated light and electron microscopy. Methods Cell Biol 79:575-591

Sterio DC (1984) The unbiased estimation of number and sizes of arbitrary particles using the disector. J Microsc 134:127-136

Tomás M, Marin MP, Portoles M, Megias L, Gomez-Lechon MJ, Renau-Piqueras J (2007) Ethanol affects calmodulin and the calmodulin-binding proteins neuronal nitric oxide synthase and $\alpha$ II-spectrin ( $\alpha$-fodrin) in the nucleus of growing and differentiated rat astrocytes in primary culture. Toxicol In Vitro 21:1039-1049

Touret N, Paroutis P, Terebiznik M, Harrison RE, Trombetta S, Pypaert M, Chow C, Jiang A, Shaw J, Yip C, Moore H-P, Van der Wel N, Houben D, Peters PJ, De Chastellier C, Mellman I, Grinstein S (2005) Quantitative and dynamic assessment of the contribution of the ER to phagosome formation. Cell 123:157170

Vancova M, Nebesarova J, Grubhoffer L (2005) Lectin-binding characteristics of Lyme borreliosis spirochete Borrelia burgdorferi sensu stricto. Folia Microbiol 50:229-238

Vanhecke D, Studer D, Ochs M (2007) Stereology meets electron tomography: towards quantitative $3 \mathrm{D}$ electron microscopy. J Struct Biol 159:443-450

Vasile E, Oka T, Ericsson M, Nakamura N, Krieger M (2006) IntraGolgi distribution of the conserved oligomeric Golgi (COG) complex. Exp Cell Res 312:3132-3141

Watt SA, Kimber WA, Fleming IN, Leslie NR, Downes CP, Lucocq JM (2004) Detection of novel intracellular agonist responsive pools of phosphatidylinositol 3, 4-bisphosphate using the TAPP1 pleckstrin homology domain in immunoelectron microscopy. Biochem J 377:653-663

Welsch S, Habermann A, Jager S, Muller B, Krijnse-Locker J, Krausslich H-G (2006) Ultrastructural analysis of ESCRT proteins suggests a role for endosome-associated tubular-vesicular membranes in ESCRT function. Traffic 7:1551-1566

Wessendorf MW, Wang H, Schnell SA (2004) Statistical methods for in situ hybridization: identification of autoradiographically labelled cells and structures. J Microsc 215:50-61

Wilczynski GM, Konopacki FA, Wilczek E, Lasiecka Z, Gorlewicz A, Michaluk P, Wawrzyniak M, Malinowska M, Okulski P, Kolodziej LR, Konopka W, Duniec K, Mioduszewska B, Nikolaev E, Walczak A, Owczarek D, Gorecki DC, Zuschratter W, Ottersen OP, Kaczmarek L (2008) Important role of matrix metalloproteinase 9 in epileptogenesis. J Cell Biol 180:10211035

Wilson BS, Steinberg SL, Liederman L et al (2004) Markers for detergent-resistant lipid rafts occupy distinct and dynamic domains in native membranes. Mol Biol Cell 15:2580-2592

Wu J, Wang Y, Xiao W, Meyer KB, Schmidt KM, Morris RE, Degen SJ, La Barbera AR (2004) Assessment of recombinant porcine follicle-stimulating hormone receptor using a novel polyclonal ectodomain antibody. Endocr Res 30:269-285

Young FM, Thomson C, Metcalf JS, Lucocq JM, Codd GA (2005) Immunogold localization of microcystins in cryosectioned cells of Microcystis. J Struct Biol 151:208-214

Zhang L-H, McManus DP, Sunderland P, Lu X-M, Ye J-J, Loukas A, Jones MK (2005) The cellular distribution and stage-specific expression of two dynein light chains from the human blood fluke Schistosoma japonicum. Int J Biochem Cell Biol 37:15111524

Zuber C, Fan J, Guhl B, Roth J (2005) Applications of immunogold labelling in ultrastructural pathology. Ultrastruct Pathol 29:319330 